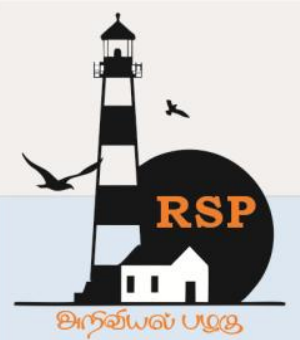

INTERNATIONAL RESEARCH JOURNAL ON ADVANCED SCIENCE HUB

\title{
Wireless Transceiver Module HC-12 based Automatic Water-level Monitoring and Control System
}

K P Deshmukh

Associate Professor, Department of Electronics, Shri Shivaji Mahavidyalaya, Barshi - 413411, Maharashtra, India.

kpdeshmukh@rediffmail.com

\begin{abstract}
This paper proposes a wireless approach for water-level monitoring and control-system using standard wireless transceiver module $\mathrm{HC}-12$ in a legal frequency band of $433.4 \mathrm{MHz}$ to $473.0 \mathrm{MHz}$ supported with hundred communication channels. The monitoring and control range is up to 500 meters in open-air and about 100 meters indoor. The microcontroller Arduino ATMEGA328 is used as monitoring and controlling device. The module is set initially for the baud-rate of 9600, $20 \mathrm{dBm}$ transmitting power, -112 dBm receiving sensitivity, $445 \mathrm{MHz}$ transmission frequency and channel number 50. All the modules in the network are set to the same parametric values to achieve synchronized communication. The controlling program collects the information regarding water-levels in different tanks and initiates control action as to start or stop the water pumping motor. It also displays the status of the water levels in different tanks. The design is tested for the household purpose. To achieve long range communications, the LORA modules could be used but for higher cost.
\end{abstract}

Keywords:wireless, transceiver, module, HC-12, automatic, water-level, monitoring, control system

\section{Introduction}

Wired type and IOT-based water-level monitoring and control system are already in the market. But the major drawback of wired-type is large wiring network from overhead-tank and ground-tank to the control system. The power losses in the wiring, electromagnetic interference, aging of the wiring, wear and tear, and cost. The problem is multiplied if building is multistore. On the other hand, the IOT-based systems require internet connectivity. To counteract these drawbacks we propose a wireless approach. The advantages of wirelessapproach are, the wiring network is avoided that results in lowering the cost, reducing electromagnetic interference, no wear and tear, no aging effect, no power-loss and hence almost no maintenance. Number of low-cost wireless modules have already populated the market. But they suffer drawbacks like; networking is not possible, very less communication distance of the order of 10 to 20 meters, lesser receiving sensitivity, low transmitting power etc. We selected HC-12 wireless serial communication transceiver module on the basis of cost, networking capability, data security, large communication distance of over 100 meters, and high receiver sensitivity. $\mathrm{HC}-12$ module is available at a cost of about Rs.150/- with properly matched spring antenna. The communication distance is enhanced if SMA antenna is used. Although, the datasheets of HC-12 module claim 1 $\mathrm{KM}$ wireless communication distance in open air but practically we observed a communication distance of about 300 meters in robust environment. The indoor communication distance of less than 100 meters is sufficient even for 10 to 15 multistore building. The basic block diagram of the wireless system is as shown in fig.1.The 
www.rspsciencehub.com

message includes an alphanumeric password. The module matching the password will only respond to the control unit and share information regarding water-level in the tank. Based on water-level information from the tank-units, the control unit

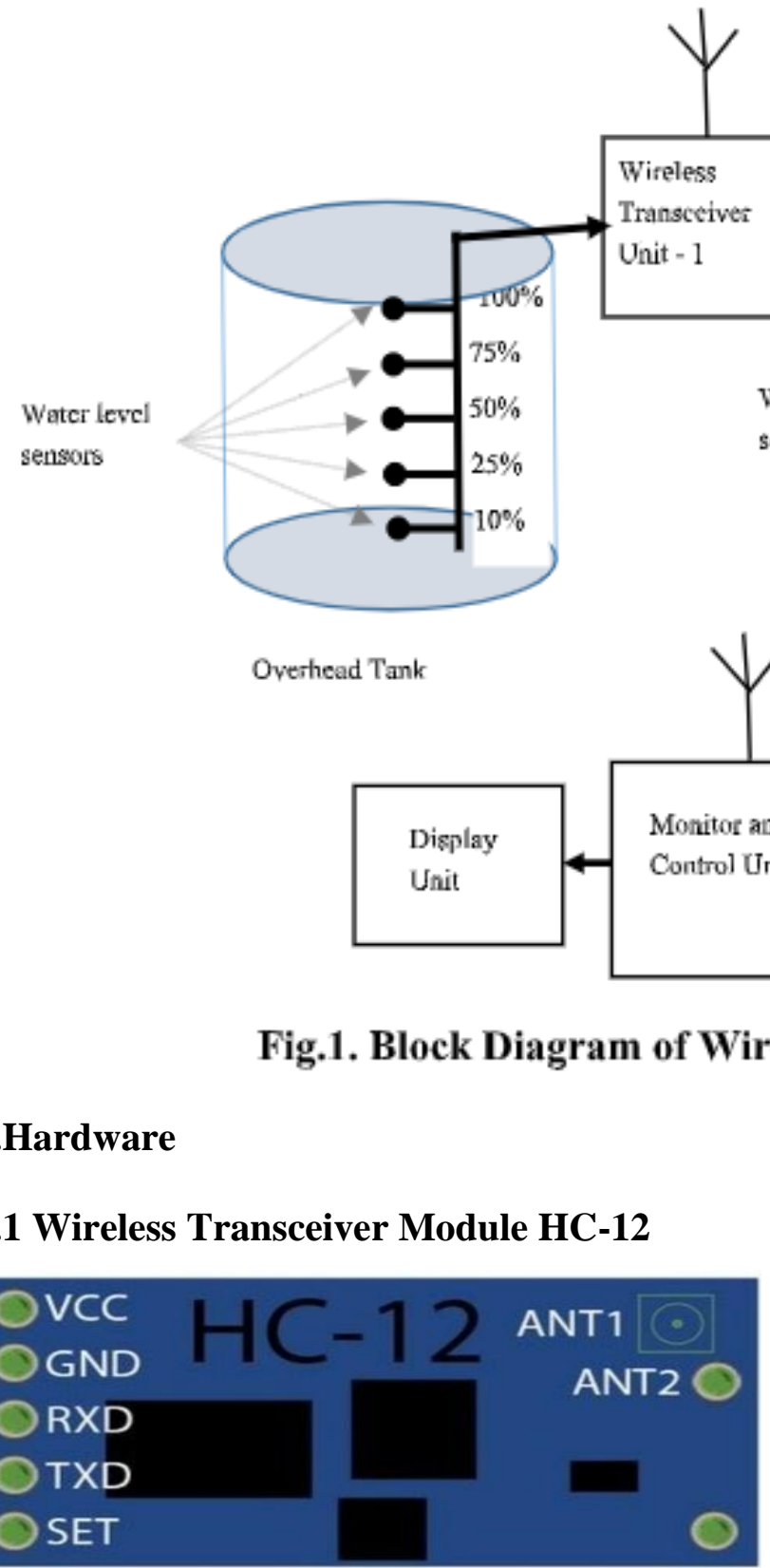

Fig.2. show the image of wireless module $\mathrm{HC}$ 12.

HC-12 is a wireless serial port communication module used in variety of applications like wireless sensor, building security, wireless robot control, PC wireless networking etc.The figure shows the image of wireless module HC-12. HC12 is a wireless serial port communication module used in variety of applications like wireless sensor,
Volume 02 Issue 10 October 2020

initiates control action to start or stop the pumping motor. The chaos of communication between modules is avoided through proper password setting which is the basis of the standalone wireless system.[1-4]

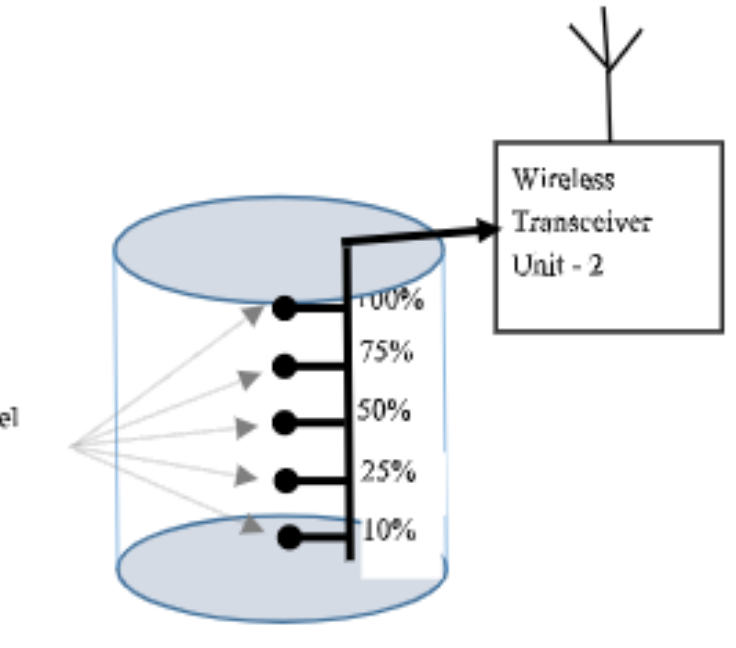

Underground Tenk

Control Signal to

Start/Stop water

puinp 
set reply, version, sleep mode, default values etc.

\subsection{Arduino Uno (ATmega328) board}

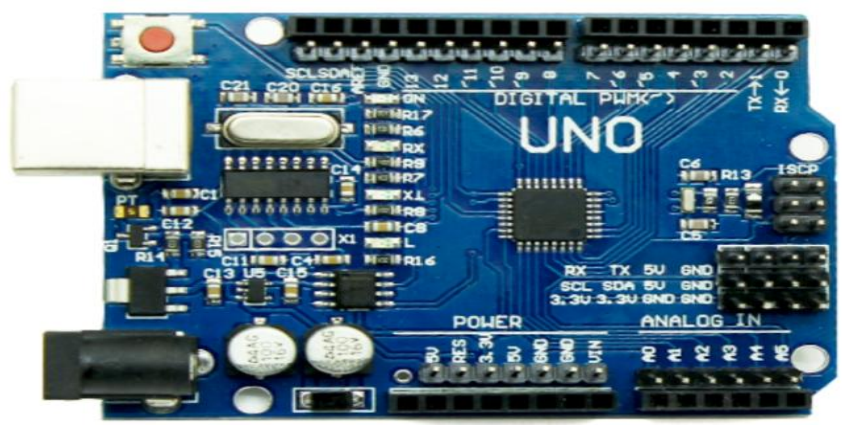

Fig.3. Arduino Uno (ATmega328) board
Circuit Serial programming. It supports $2 \mathrm{~KB}$ SRAM, $32 \mathrm{~KB}$ flash memory, $1 \mathrm{~KB}$ EEPROM and runs on $16 \mathrm{MHz}$ clock. The software control is accomplished by the use of $\mathrm{C}$ functions like pinMode( ), digitalRead( ), digitalWrite( ), analogRead( ) etc. Many other technical specifications could be obtained from technical manual available from Arduino official website.

\section{Connection Diagram}

The connection diagram for wireless control system is shown in fig.4. The baud-rate, power

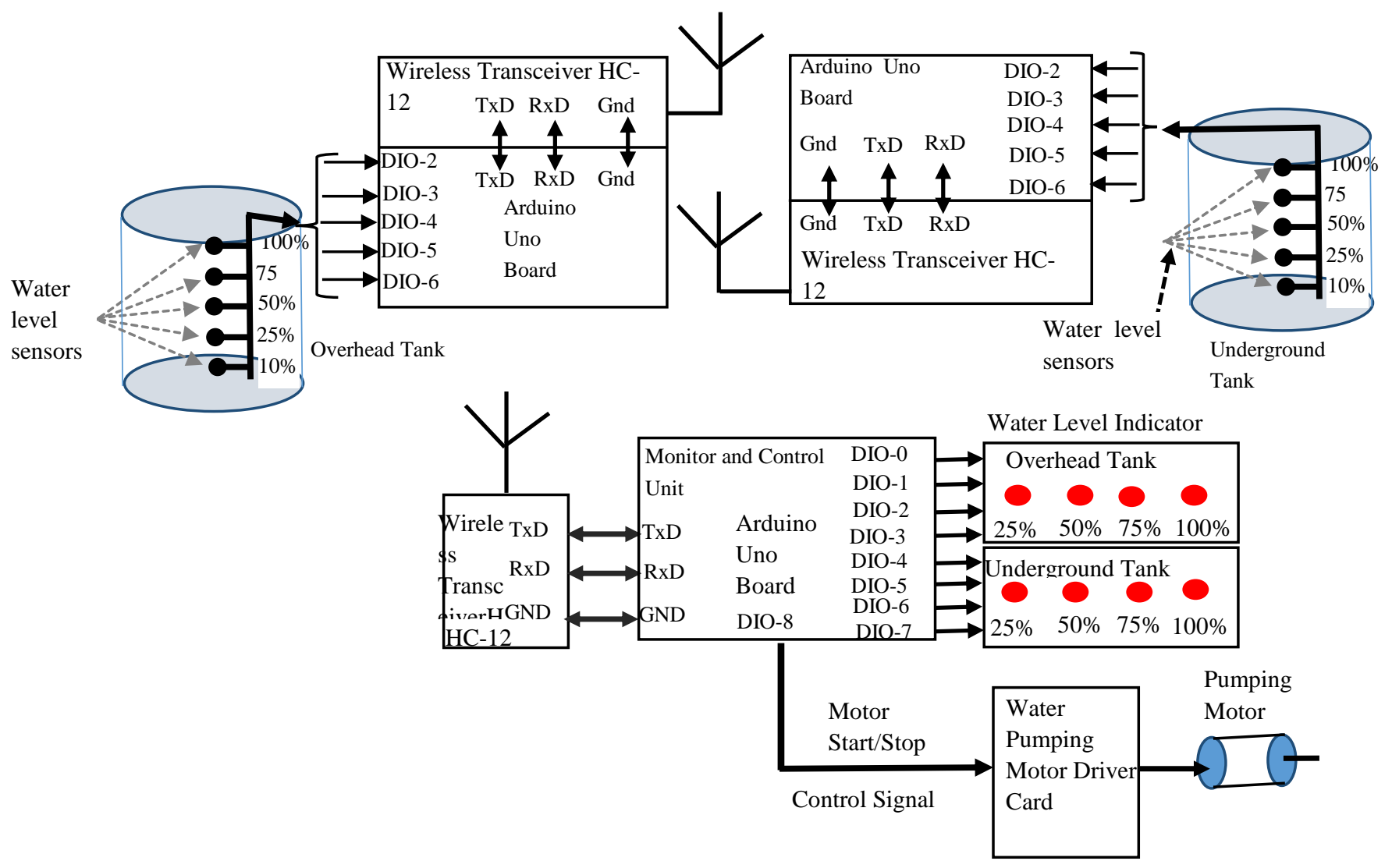

Fig.4. Connection Diagram of Wireless Control System

Fig.3. shows image of Arduino-Uno board from Microchip Inc. It has 14-digital input-output pins (DIO-0 to DIO-13), one analog reference pin $\mathrm{A}_{\text {ref }}$. Six DIO pins can also be used as PWM output. DIO-0 and DIO-1 are receive data and transmit data pins for serial communication. There are six analog input pins $A_{0}$ to $A_{5}$, with analog voltage range of 0 to $+5 \mathrm{~V}$. It includes a 10-bit $A$ to $D$ converter. A hardware RESET switch to manually RESET the microcontroller. The board support In- output, receiver sensitivity, channel number selection, transparency settings for $\mathrm{HC}-12$ are initialized by connecting $\mathrm{HC}-12$ module to $\mathrm{PC}$ or laptop through FTDI module. All the HC-12 modules are set to same parametric values like; $9600 \mathrm{bps}, 100 \mathrm{~mW},-117 \mathrm{dBm}, 50$, FU3 etc. This is mandatory setting for all the wireless modules in the same network. The wireless module connected to the water tanks will supply water-level information whenever required by the control unit. 


\section{www.rspsciencehub.com}

For this, the control unit sends the message to water-tank units, along with the alphanumeric password. The water-tank unit, whose password matches, will respond to the control unit, by sending the water-level information. In this manner, the half-duplex communication is achieved between control unit and water-tank units. This water-level information could be collected periodically after every half-an hour, as per requirements.

\section{Software}

The flowchart for the water-tank unit and control unit are shown in fig. 3 and fig.4. The flowcharts of the wireless system are selfexplanatory. The code is written in embedded-C, using Arduino IDE.

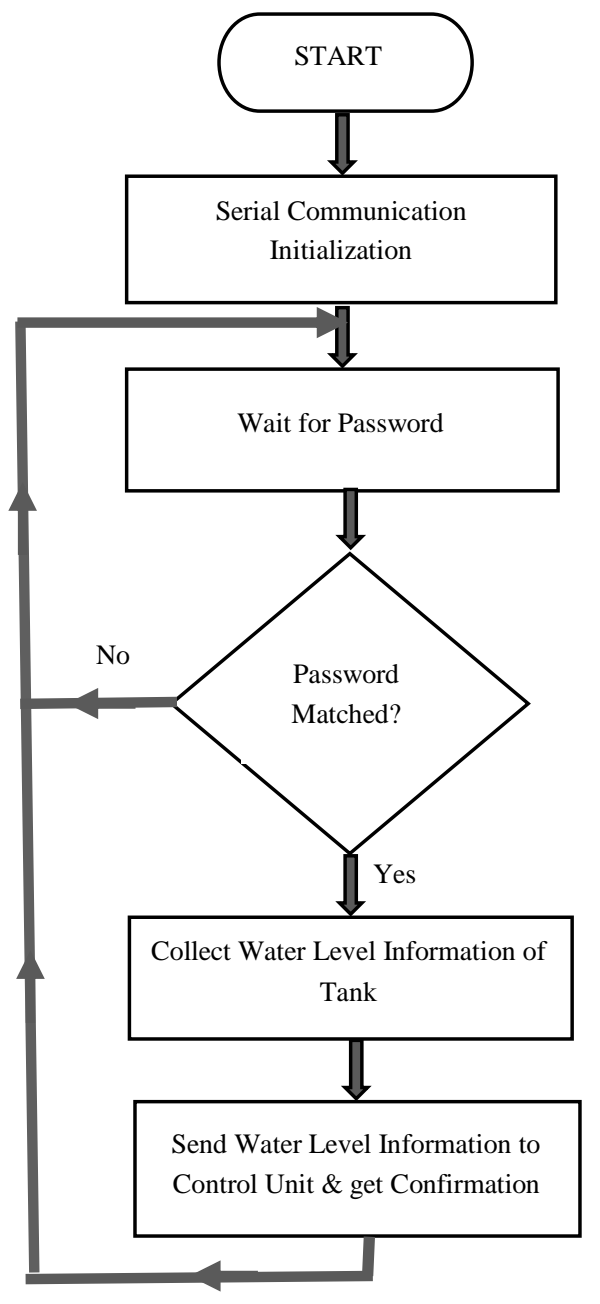

Fig.3. Flowchart of Water Tank Circuit
Volume 02 Issue 10 October 2020

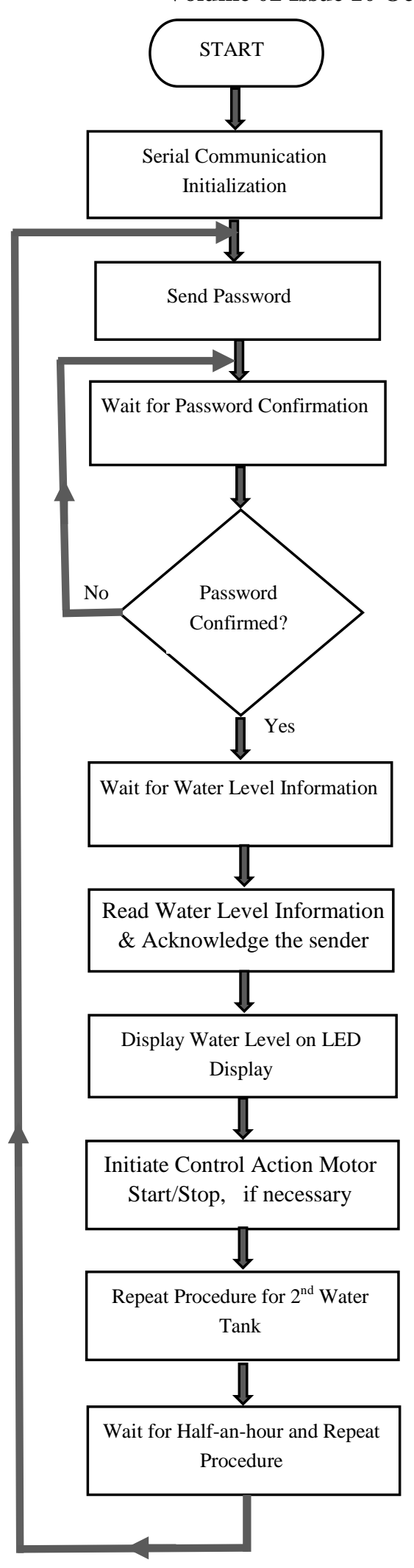

Fig.4. Flowchart of Monitoring and Control System

\section{Conclusions: -}

The wireless system discussed in the paper 


\section{www.rspsciencehub.com}

works in robust environment over a distance of about 100 meters for indoor application and about 300 meters in open air. It is cost-effective, rugged and reliable as compared to the wired and IOTbased systems requiring internet connectivity. Hence the wireless system is highly recommended for house-hold purpose.

\section{Results: -}

The true range of wireless communication varies with environmental conditions. The wireless system operates as per the requirements. It is a stand-alone system feasible in urban as well as rural areas equally, without the issue of connectivity or wiring network.

\section{Acknowledgement: -}

The author likes to thank and acknowledge the support from Department of Electronics, Shri Shivaji Mahavidyalaya, Barshi-413411, for providing laboratory facilities, electronic material and library access.

\section{References}

\section{Journals}

[1].SantraM., BiswasS. et al. (2017). Smart Wireless Water Level Monitoring and Pump Controlling System, Int. Jour. Adv. Sci. Res. Engg., vol. 03, issue 4.

[2].DebS., ChakrabortyP. et al. (2018). Wireless Pump Control with Water Level Monitoring System, Int. Indo-Iranian Jour. Sci. Reser., vol.2, issue-2, pp. 5-10.

[3].Md. MominS A, RoyP. et al. (2016). Construction of Digital Water Level Indicator and Automatic Pump Controlling System, Int. Jour. Reser., vol.03, issue-12..

[4].Ms. AshaT, Ms. SrijaV. (2020). Design and Implementation of Wireless Based Water Level Monitoring System using Arduino and Bluetooth, Int. Resear. Jour. Engg. Tech., vol.7, issue-01.

[5].GuptaN., KumarS. et al. (2016). Wireless Water Level Controller using Zigbee, IJLEMAS, vol.V, issue-IV.

[6].Nishmitha, ShettyS. et al. (2019). Water Tank Monitoring System, IJERT, special issue 2, conf. proc., ISSN:2278-0181.
Volume 02 Issue 10 October 2020

[7].MittalV. (2017). Automatic Water Level Controller, IJSR, vol.6, issue-5, ISSN: 23197064.

[8].MounikaJ., Siva Kumar ReddyN. (2016). Water Monitoring System based on GSM, IARJSET, vol.3, issue-7, ISSN: 2393-8021. 